\title{
Adolescent insight within the working alliance: A bridge between diagnostic and psychotherapeutic processes
}

This article was published in the following Dove Press journal:

Adolescent Health, Medicine and Therapeutics

7 July 2010

Number of times this article has been viewed

\author{
Michela Gatta' \\ Andrea Spoto ${ }^{2}$ \\ Paolo Testa ${ }^{3}$ \\ Lorenza Svanellini ${ }^{3}$ \\ Jessica Lai ${ }^{3}$ \\ Maurizio Salis ${ }^{3}$ \\ Maxim De Sauma ${ }^{4}$ \\ Pier Antonio Battistella ${ }^{3}$ \\ 'Department of Paediatrics, \\ 2Department of General Psychology, \\ ${ }^{3}$ Neuropsychiatric Unit for Children \\ and Adolescents, Azienda ULSS 16 , \\ Padua, Italy; ${ }^{4}$ Brent Centre For Young \\ People, London, UK
}

\begin{abstract}
In the literature it has been stressed how important it is during consultation interviews to evaluate an adolescent's insight with a view to starting psychotherapeutic work. Motivated adolescents bring to the therapeutic effort a real, interiorized request for help and can start moving towards a change. The aim of this study is to assess the insight of adolescents with mental disorders during the diagnostic process and to analyze how this relates to further therapeutic compliance and clinical evolution. The study included 55 adolescents, 35 males and 20 females aged between 11 and 19 years, who were advised to receive psychotherapeutic treatment after a diagnostic procedure. Patient insight was assessed at the beginning and end of the diagnostic process. Then, six months later, a clinical interview assessed their therapeutic compliance and clinical evolution. The findings suggest that insight is important when working with adolescents because it influences both therapeutic compliance and clinical outcome.
\end{abstract}

Keywords: adolescence, insight, therapeutic alliance, therapeutic compliance, outcome

\section{Introduction}

The therapeutic alliance is a strong predictor of outcome in individual psychotherapy across diverse treatment orientations and modalities, and pathologies, whether the patients are adults ${ }^{1-5}$ or adolescents. ${ }^{6-9}$

When it comes to psychotherapy with adolescents, several works highlight the need to establish an alliance with the adolescent patient right from the first steps of the consultation, ${ }^{7-10}$ while other authors emphasize the importance of taking similar action with members of their families in the belief that, in order to deal with young patients, it is important to deal with their parents too, ${ }^{11-13}$ particularly when young patients have behavioral disorders. ${ }^{14-16}$

To contextualize insight within the therapeutic alliance, if we consider the the therapeutic alliance as an interaction between two poles (the therapist and the patient, each with their own intrapersonal and interpersonal features) within a space defined by the setting, insight is one of the patient's intrapersonal characteristics. Insight is often the most commonly used concept to understand why people with mental illness do or do not follow treatment suggestions. This has been widely studied in relation to severe psychopathologies, including psychosis or mood disorders, mainly in adults. ${ }^{17,18}$ The majority of studies support the assumption that insight is associated with adherence during the treatment phase and correlates with better long-term functioning. ${ }^{19}$

With regard to adolescent patients, some authors ${ }^{20,21}$ have stressed how important it is during consultation interviews to evaluate insight with a view to starting psychotherapeutic work. Motivated adolescents bring to the therapeutic effort a real, 
internalized request for help and they can start moving towards a change. Clinical practice shows that adolescents often resist feeling and manifesting the need for help. Even when their request for help is openly expressed, they are frequently still very distrustful of adults, so they strongly resist establishing a relationship of dependence with the psychotherapist, who is seen as an adult figure. ${ }^{20,22}$ Patient insight during therapeutic sessions has been much studied, ${ }^{18,23,24}$ whereas patient insight associated with the diagnostic process has attracted less attention in the scientific literature. ${ }^{20,25,26}$ Alongside the theory, there have been clinical experiences, and our past pilot study on the therapeutic compliance of adolescents ${ }^{27}$ showed that most adolescents did not follow the recommendation to undergo psychotherapy after the diagnostic process. Compliant and noncompliant adolescents differed in terms of the self-consciousness expressed during the diagnostic process. The more motivated adolescents started therapy, while most of the unmotivated adolescents did not.

These results and other findings in the literature showing that an early alliance has proved a better predictor of outcome than the alliance averaged across sessions or measured midway or late in the treatment process, ${ }^{28}$ that insight is one of the relevant factors affecting compliance and outcome, ${ }^{19,29}$ and that future research should address the complex interactions between interventions, a patient's level of functioning, helping alliance and outcome, ${ }^{30}$ and prompted us to consider some strategies to adopt in clinical practice to augment an adolescent's motivation to comply with the recommendations for therapy at the end of the diagnostic process. We devised a diagnostic protocol that pays particular attention to aspects related to the patient's insight when considering their psychiatric diagnosis and psychopathologic investigation.

Being aware of the complexity of the concept of insight and its evaluation, we agree with the approach taken by Markova and Berrios ${ }^{31}$ who developed the Insight Scale (IS), based on the hypothesis that insight can be seen as a subcategory of self-consciousness rather then as an independent aspect of the psychiatric disorder. This places the emphasis not only on a patient's awareness of their illness, but also on how much this influences their interaction with the environment. This is valid in general, but even more so in developmental age, when individuals are still dependent on adults, and can only be considered in terms of their relationship with the environment.

\section{Methods}

\section{Participants}

The study included 60 adolescents (38 males and 22 females) aged between 11 and 19 years, referred consecutively to our service over one year for a clinical evaluation and recommended for psychotherapy. Five were excluded because they and/or their parents did not consent to participation in the study (three males, aged 12, 13, and 16 years, with anxiety, behavioral disorders, and an impulsive personality disorder, respectively, and two females, aged 15 and 17 years, with an eating disorder and an impulsive personality disorder, respectively).

The final sample therefore consisted of 55 adolescents, 35 males (64\%) and 20 females (36\%), aged between 11 and 19 years. They were divided into three age groups: 11-13 years (16 cases, 29\%), 14-16 years (23 cases, 42\%), and 17-19 years (16 cases, 29\%). With regard to education, $5.5 \%$ were attending primary school, $38.2 \%$ were at high school, $54.5 \%$ went to college, and $1.8 \%$ had left school. The family's formal education was low for $16.4 \%$ of the adolescents, medium for $63.6 \%$, and high for $20 \%$. Mode of presentation was at the adolescent's spontaneous request for a psychodiagnostic consultation in $38.2 \%$ of cases, and through referral in $61.2 \%$. Observed frequencies and percentages distribution by gender, adolescents' and parents' age groups, psychiatric diagnosis, adolescents' insight, and parents' educational level are reported in the Table 1.

We screened our adolescent patients with the Wechsler Intelligent Scale for Children Revised (WISC-R) ${ }^{32}$ and Wechsler Adult Intelligence Scale Revised (WAIS-R) ${ }^{33}$ to exclude those whose Intelligent Quotient (IQ) was $<70$, but included patients with a borderline IQ $(70 \leq \mathrm{IQ} \leq 80)$ when it was the only diagnosis. Other exclusion criteria were age below 11 years, acute psychotic states or psychiatric conditions requiring hospitalization and/or pharmacologic treatment, and known organic diseases associated with mental disorders.

\section{Diagnostic processes}

The neuropsychiatric consultation, the aim of which was to formulate a psychiatric diagnosis and to evaluate the patient's insight, was organized with separate diagnostic interviews with the adolescents and their parents conducted by a child and adolescent neuropsychiatrist and a trained psychodynamic therapist. The consultation was arranged according to the following protocol. For the consultation between the adolescent and the neuropsychiatrist), there was a first interview for acceptance, followed by two clinical interviews with any required tests, a final interview (to communicate the diagnosis and therapeutic recommendations), and a semistructured interview focusing on discussion of the recommended psychotherapy. In parallel, there was a consultation between the 
Table I Observed frequencies distribution by gender, adolescents' and parents' age groups, psychiatric diagnosis, adolescents' insight, and parents' educational level

\begin{tabular}{|c|c|c|c|}
\hline & & $\mathbf{n}$ & $\%$ \\
\hline \multirow[t]{3}{*}{ Gender } & Male & 35 & 63.6 \\
\hline & Female & 20 & 36.4 \\
\hline & Total & 55 & 100 \\
\hline \multirow[t]{4}{*}{ Age group } & $11-13$ years & 16 & 29.1 \\
\hline & $14-16$ years & 23 & 41.8 \\
\hline & $17-19$ years & 16 & 29.1 \\
\hline & Total & 55 & 100 \\
\hline \multirow[t]{5}{*}{ Insight (first diagnostic interview) } & & & 32.7 \\
\hline & Motivated & 18 & 41.8 \\
\hline & Indifferent & 23 & 25.5 \\
\hline & Resistant & 14 & \\
\hline & Total & 55 & 100 \\
\hline \multirow[t]{10}{*}{ Diagnosis (ICD I0) } & Not mental disorder according to ICD-I0 & I & 1.8 \\
\hline & Neurotic, stress and somatoform disorder & 18 & 32.7 \\
\hline & Affective disorder & 4 & 7.3 \\
\hline & Psychotic disorder & 3 & 5.5 \\
\hline & Personality disorder & 13 & 23.6 \\
\hline & Behavioral and emotional disorder & 8 & 14.5 \\
\hline & Eating disorder & 3 & 5.5 \\
\hline & Borderline $\mathrm{IQ} 70 \leq \mathrm{IQ} \leq 80$ & 2 & 3.6 \\
\hline & Comorbidity (personality disorder + anxiety or mood disorder) & 3 & 5.5 \\
\hline & Total & 55 & 100 \\
\hline \multirow[t]{4}{*}{ Insight (last diagnostic interview) } & Motivated & 36 & 65.5 \\
\hline & Indifferent & II & 20 \\
\hline & Resistant & 8 & 14.5 \\
\hline & Total & 100 & 100 \\
\hline \multirow[t]{5}{*}{ Mother (age group) } & $\leq 45$ years & 10 & 18.2 \\
\hline & $46-50$ years & 20 & 36.4 \\
\hline & $5 \mathrm{I}-55$ years & 13 & 23.6 \\
\hline & $>55$ years & 12 & 21.8 \\
\hline & Total & 100 & 100 \\
\hline \multirow[t]{5}{*}{ Father (age group) } & $\leq 45$ years & 5 & 9.1 \\
\hline & $46-50$ years & 16 & 29.1 \\
\hline & $5 \mathrm{I}-55$ years & 18 & 32.7 \\
\hline & $>55$ years & 16 & 29.1 \\
\hline & Total & 100 & 100 \\
\hline \multirow[t]{4}{*}{ Educational level } & low & 9 & 16.4 \\
\hline & medium & 35 & 63.6 \\
\hline & high & II & 20 \\
\hline & Total & 55 & 100 \\
\hline
\end{tabular}

parents and a psychologist assisted by an observer, comprising a first interview for acceptance, two clinical interviews, collection of the adolescent's clinical history, a final interview (to communicate the diagnosis and therapeutic recommendations), giving an explanation of the findings, and obtaining the adolescent's and parents' signed informed consent.

The final meeting with the adolescent aimed to discuss the treatment recommendations. The purpose of this difference vis-à-vis the usual diagnostic protocol (when the diagnosis is communicated and treatment is recommended at the last meeting with the adolescent) was to provide information about psychotherapy (what it is, how it works, what it is useful for) and to prompt the adolescent to ask questions, voice any doubts, fantasies and/or anxieties about psychotherapy, and to talk about them with the specialist. The assumption was that giving them an opportunity to bring up these issues and receive information might help to improve their insight and motivate them to accept therapy. The diagnostic processes were implemented by six clinicians, three child and adolescent neuropsychiatrists, and three psychologists, who were matched differently at each consultation, and attended by three observers who were also combined differently at each consultation. 
The psychiatric diagnosis was formulated using the ICD $10 .{ }^{34}$ The adolescent's insight was evaluated at baseline (after the first interview) and at the end of the diagnostic process (after the last interview). To measure the adolescent's insight at the start and end of the diagnostic process, we used the validated Italian version of the IS..$^{31,35,36}$ This eight-item self-report scale is designed to be sensitive to changes in levels of insight, and captures each of three widely accepted dimensions of insight, ie, perceived need for treatment, awareness of illness, and relabeling of symptoms as pathologic. Higher scores indicate greater levels of insight. The psychometric properties of the IS are excellent and it is widely used in psychiatry research. As suggested in the guidebook, ${ }^{37}$ the analysis considered the items in groups A and B. The minimum score possible was 22 and the maximum was 37 , and three groups of scores or ratings were identified, ie, $\leq 25,26-31$, and 32-37, corresponding to three different insight levels, that we named according to a sorting of adolescent characterization as motivated (high IS, 32-37), indifferent (medium IS, 26-31) and resistant (low IS, <26).

The clinician who interviewed the parents was assisted by a neutral observer (a psychodynamic-oriented trainer psychologist), who evaluated the parents' attitudes during the last sessions. The aim was to consider another variable, ie, parental capacity to collaborate in the adolescent's treatment, using the Working Alliance Inventory, Observer Short version (WAI-O-S) translated into the Italian language. ${ }^{38-40}$ This scale consists of 12 items, 10 positively worded and two negatively worded, rated on a seven-point Likert-type scale. The items are divided into three subscales of four items each. The subscales, based on Bordin's working alliance theory, ${ }^{41}$ are "goal" (agreement about goals of therapy; eg, "The client and therapist have established a good understanding of the changes that would be good for the client"), "task" (agreement about the tasks of the therapy; eg, "There is agreement on what is important for the client to work on"), and "bond" (the bond between the client and therapist; eg, "There is mutual trust between the client and therapist"). The WAI-O-S has been previously shown to have good reliability $(r=0.81$, L. Gelfand and R. DeRubeis, unpublished manuscript), and research has also shown strong support for the reliability of the WAI scales in general, as well as some support for their validity. ${ }^{42,43}$ Given our purpose, ie, to evaluate parental capacity to collaborate with the adolescent's treatment, we selected the WAI-O-S task subscale (items 1,2,8, and 12), to be filled in during diagnostic interview (the last one). Ranging from a minimum of 0 to a maximum of 28, the ratings were coded as "uncollaborative" parents (scoring 0-14) and "collaborative" parents (15-28). The observer was blinded to the IS ratings.
A clinical history form was completed for each adolescent to collect personal details and information on their family, psychosocial situation, and clinical aspects.

The treatment that the adolescents received was individual psychodynamic-oriented short psychotherapy organized in weekly or fortnightly sessions and conducted by trained psychologists (not the clinicians who performed the first evaluations). This form of treatment, where the psychotherapist is much more actively involved than in classical psychoanalytic psychotherapy, focuses directly on the patient's actual feelings and emotions, considering specific elements such as an adolescent's self-representations, object relations, and issues regarding the processes of separation and individualisation. ${ }^{44,45}$ There were six psychologists in total, each of whom took a given individual into their care, depending on their agendas at the time. Data were collected on the patient's therapeutic compliance and clinical outcome at a follow-up clinical interview six months after the last diagnostic session. These data were based on the changes in symptoms after six months of therapy identified by the specialist who had first diagnosed the case. Item 2 of the Italian version of the Clinical Global Impressions $(\mathrm{CGI})^{46}$ scale, ie, the one relating to global improvement, was completed for each adolescent, and patients were considered as having become better when their score was 1-2, remained unchanged if the score was $3-5$, and become worse when the score was 6-7. The clinician was blinded to the adolescent's IS ratings and to the neutral observer's considerations concerning parental collaboration (WAI-O-S task subscale).

\section{Statistical analysis}

We considered the "insight" variable in relation to gender (male/female), age (11-13, 14-16, or 17-19 years), the family's formal education, assessed from matching each parent's schooling (low, middle, high), mode of presentation (voluntary, through referral), parental collaboration (collaborative or uncollaborative parents), diagnosis (ICD 10), therapeutic compliance (receiving therapy, dropped out, never started therapy), and six-month follow-up (clinically better, unchanged, worse). The data are expressed as frequencies and percentages. Variables are expressed using nominal and ordinal scales. Cross-tabulations were analyzed using the chi-square test, considering $P<0.05$ as significant. The analyses were performed using SPSS rel. 14.

\section{Results and discussion}

The insight measure recorded at the first interview showed that only one-third of the sample (33\%) agreed to the diagnostic process, suggesting that the adolescent's motivation 
should be a goal of any intervention rather than taken for granted. This situation is confirmed in the literature ${ }^{20,47}$ and consistent with the findings concerning mode of presentation, which showed that nearly two-thirds of the adolescents came because they had been referred by others (medical practitioners, hospital staff, social services, legal authorities), and even when a spontaneous presentation had come, it was not because they themselves had wanted to, but because the visit had been requested by their parents and not by the adolescents themselves.

We tried to change this initial situation by adjusting the diagnostic process, and the last interview in particular, in an attempt to create a relationship with the patients and help them to become aware of their disease and develop the desire to change. We consider insight as being closely connected with self-consciousness and motivation to receive treatment. Recognizing and admitting a state of unease is the first step towards dealing with it. Being worried about it means being in touch with the anxiety caused by this condition, and the desire to change depends on this awareness. Being able to observe and describe oneself means being in touch with one's own inner world and being able to tolerate one's anxiety.

We tried to determine whether the "initial insight" variable differed significantly by gender, age, or the parents' formal education. At the beginning of the diagnostic process there were some differences in the sample as regards gender $\left(\chi_{2}^{2}=7.26, P<0.05\right)$ and the parents' formal education $\left(\chi_{4}^{2}=11.20, P<0.05\right)$, but no differences in terms of adolescent age. The correlation analysis indicated that females have a better insight about their condition than males ( $\mathrm{rho}=-0.28$, $P<0.05$ ), while no significant correlation emerged between their insight and the parents' formal education. The same analyses were performed at the end of the diagnostic process and the results indicated that there was still no difference in insight by gender and age, while a significant difference was confirmed between cases from different sociocultural environments $\left(\chi_{4}^{2}=13.80, P<0.05\right)$. Figures 1 and 2 show the different insight levels at the start and end of the diagnostic procedure by age and gender, respectively.

This result suggests that environmental aspects (such as the parents' formal education) might influence adolescent thinking capacity more than constitutional factors (eg, gender or age). It also suggests that it might be more appropriate to provide supportive rather than expressive care for people coming from more disadvantaged sociocultural backgrounds. These differences are interesting mainly from a descriptive point of view, however, and they did not affect the subsequent results found in the sample as a whole.
We focused on any significant differences in the levels of insight at the start and end of the diagnostic assessment process. Our results showed a significant difference between the measures obtained at baseline and after completing the diagnostic workup, pointing to an improved insight in the sample as a whole $(\mathrm{Z}=-3.30, P<0.05)$.

With regard to the three categories identified on the basis of the adolescents' insight at the end of the diagnostic process (post-diagnosis IS), $65.5 \%$ of the adolescents were motivated, $20 \%$ were indifferent, and $14.5 \%$ remained resistant. The adolescents in the first group could be described as individuals who recognized their disease, were worried about it, and wanted to change, whereas those in the second group admitted to and could describe their disease, but did not seem worried and tended to minimize their psychological condition, and those in the third group generally came to us only because their parents obliged them to do so, and therefore did not acknowledge their disease and said it was somebody else's fault that they were meeting with a clinician. In terms of psychic functioning modality, the difference between the indifferent and the resistant categories lies in the passive and active dimensions, respectively, characterizing their resistance to meeting a clinician.

It is worth noting that there was a more pronounced change with respect to the motivated category in the resistant group than in the indifferent group. The group that was resistant at baseline was halved in size during the diagnostic process, showing that these adolescents had a greater capacity for mobilization. Psychodynamic assessment of the adolescents and their families showed a high frequency of difficult relationships and conflictual relationships with parents among the resistant cases; the item for "presence of conflict between parents and offspring" in the family history form was ticked in $73 \%$ of cases compared with $12 \%$ of the indifferent adolescents and $16 \%$ of the motivated adolescents. This aspect could be important in influencing the adolescents' feelings about the specialist and the psychological space, suggesting that their difficult relationships with their parents were expressed by their rejection of a psychiatric consultation to which they have been brought by their parents rather than coming of their own volition. In such cases, focusing on these problematic relationships and on the separation/individualization processes might favor the adolescents' use of a psychodiagnostic space as their own, rather than as a forum in which to attack their parents. Although the resistant adolescents were evidently more oppositional, they proved more "malleable" than the indifferent adolescents, whose defenses were more 

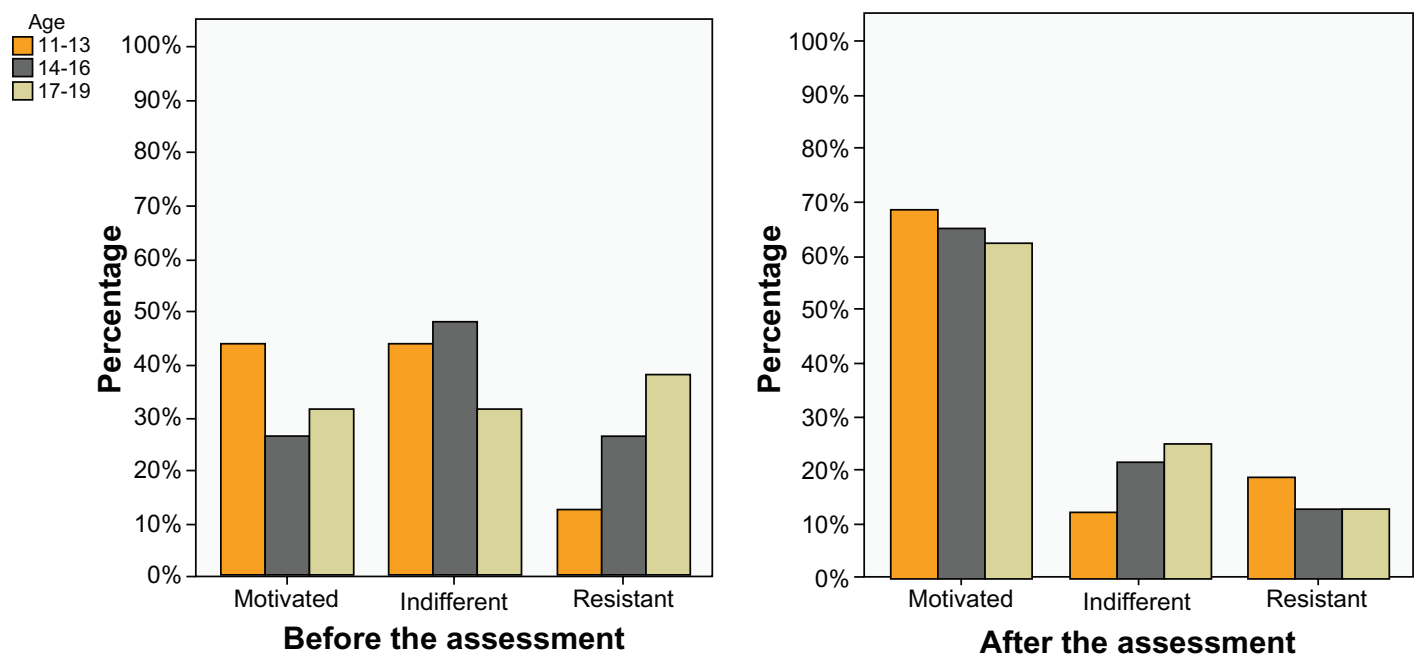

Figure I Different levels of insight before and after assessment by age.

inflexible, and their emotional distance made it difficult to establish an empathic relationship with them.

A six-month follow-up enabled us to ascertain the therapeutic compliance and clinical outcome. Eighty-four percent of the adolescents were compliant, $9 \%$ had dropped out, and 7\% had never started psychotherapy. On the basis of the CGI ratings, $62 \%$ of the adolescents had improved, $34 \%$ remained unchanged, and $4 \%$ had become worse.

We assumed that the adolescents' insight would have a significant effect on their compliance and the positive evolution of their conditions. Our results seem to confirm this hypothesis and also point to a marginal, but nonetheless important, effect of parental support on the outcome of treatment.

We first analyzed how and to what extent levels of compliance correlated with the different levels of outcome.

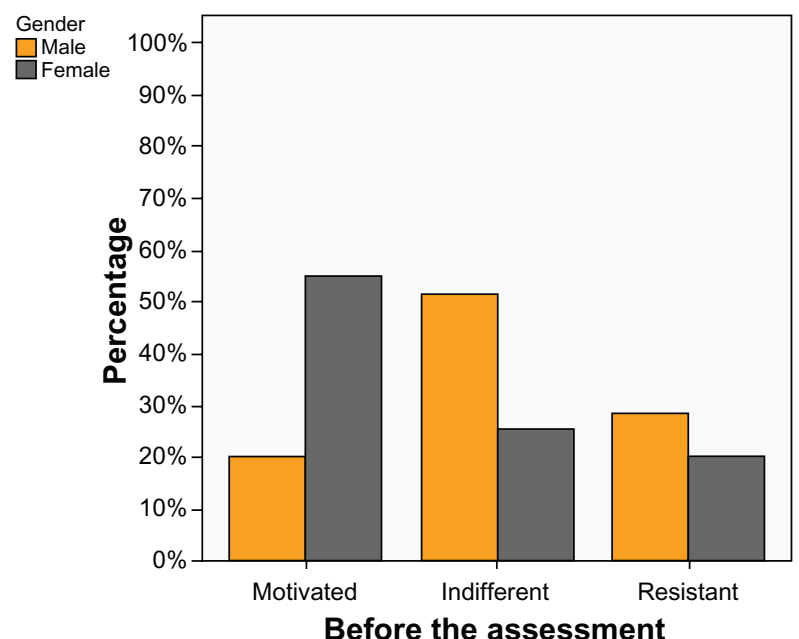

Figure 2 Different levels of insight before and after assessment by gender.
A chi-square test was used to verify the relationship between compliance and quality of outcome. The results confirmed a strong link between these two variables, and emphasized that different levels of compliance coincided with different outcomes $\left(\chi_{4}^{2}=17.32, P<0.05\right)$. Analysis of the correlation indicates that higher levels of compliance are more likely to coincide with a positive outcome ( $r h o=0.37, P<0.05)$. This suggests, therefore, that psychotherapy was effective when the adolescent routinely attended the sessions.

As mentioned above, we studied the effect of both parental collaboration and adolescents' insight on therapeutic compliance and the outcome of treatment. Parental collaboration correlated with outcome $\left(\chi_{2}^{2}=9.22, P<0.05\right)$, but did not correlate with compliance. In other words, we found that parental support had only a marginal role in

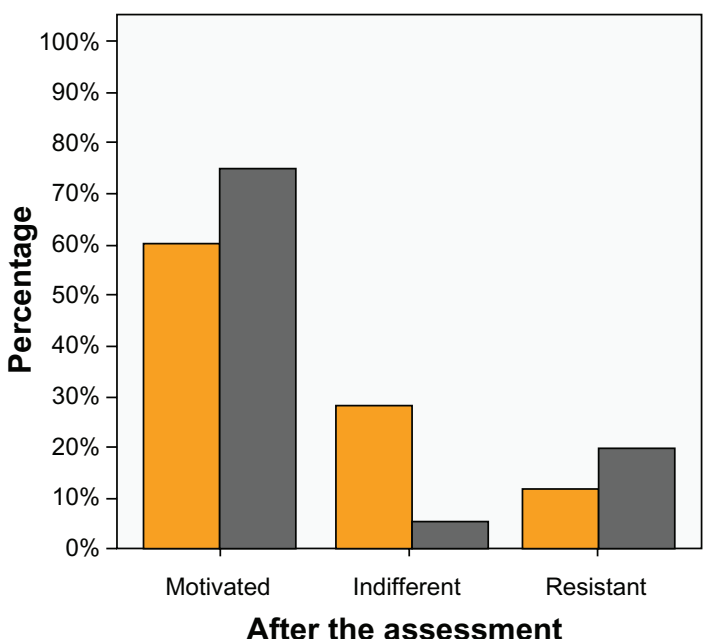


the adolescents' outcomes, their insight being what really influenced their compliance. This means that cooperation with parents is not the crucial element to explain the positive outcome, although it is an additional element that can improve the treatment.

A totally different part is played by patient insight at the end of the diagnostic phase (ie, at the start of treatment). A chi-square test was used to verify the effect of different levels of insight on the outcome of treatment, and the results indicate a crucial influence of insight on both compliance $\left(\chi_{4}^{2}=16.96, P<0.05\right)$ and outcome $\left(\chi_{4}^{2}=25.24, P<0.05\right)$. The analysis of correlation indicates that higher levels of insight coincide with an improvement in compliance (rho $=0.39, P<0.05$ ) and outcome (rho $=0.56, P<0.05$ ).

These results, together with the fact that no relationship was found between patient insight and parental collaboration, and that the relationship between insight and outcome was not affected by parental attitude, confirm that the core issue for the purposes of achieving a positive outcome in adolescents is their own motivation. Their insight can be increased during the diagnostic phase and this seems to be very useful in increasing the chances of a positive outcome.

We can therefore claim that an adolescent's insight is linked not only to their therapeutic compliance but also to the efficacy of treatment. The elements defining the patient's insight are important ingredients for building an alliance, which is an important factor for the efficacy of therapeutic process. ${ }^{1-9,48,49}$

\section{Conclusion}

From this pilot study it appears that, when working with adolescents, the most important factor leading to a positive outcome of psychotherapy is the patient's insight concerning treatment. Insight seems to have a crucial influence on the patient's therapeutic compliance and clinical outcome. We therefore believe that for an adolescent to have a collaborative family is not enough to ensure the efficacy of the therapy, and that genuine motivation is essential to overcome the patient's suffering and embark on a psychotherapeutic process.

We observed a greater degree of insight in adolescents whose parents had a better formal education, and it may be that the influence of education is associated with the theory orientation and therapeutic model adopted, ie, the psychodynamic ones.

The other result worth noting is that female adolescents were more motivated then males at the first interview, although the males showed a significant improvement after specific "motivational training". The better insight on the part of females might reflect gender being a constitutional-temperamental factor. Operatively, the application of these results might be a major investment for insight training with male adolescent patients.

\section{Disclosure}

The authors report no conflict of interest in this work.

\section{References}

1. Henry WP, Strupp HH. The therapeutic alliance as interpersonal process. In: Horvath AO, Greenberg LS, editors. The Working Alliance: Theory, Research and Practice. New York, NY: Wiley; 1994.

2. Keijsers GPJ, Scraap CPDR, Hoogduin CAL. The impact of interpersonal patient and therapist behavior on outcome in cognitive-behavior therapy A review of empirical studies. Behav Modif. 2000;24:264-297.

3. Zuroff DC, Blatt SJ. The therapeutic relationship in the brief treatment of depression: Contributions to clinical improvement and enhanced adaptive capacities. J Consult Clin Psychol. 2006;74:130-140.

4. Barber JP. Alliance predicts patients' outcome beyond in-treatment change in symptoms. J Consult Clin Psychol. 2000;68:1027-1032.

5. Newman CF, Strauss JL. When clients are untruthful. Implications for the therapeutic alliance, case conceptualization, and intervention. J Cogn Psychother. 2003;17:241-252.

6. Alvin P, Rey C, Frappier JY. Compliance thérapeutique chez l'adolescent malade chronique. Arch Pediatr. 1995;2:874-882.

7. Meeks JE, Bernet W. The Fragile Alliance: An Orientation to Psychotherapy of the Adolescent. Malabar: Krieger Publishing Co; 2001.

8. Shelef K, Diamond GM, Diamond GS, Liddle HA. Adolescent and parent alliance and treatment outcome in multidimensional family therapy. J Consult Clin Psychol. 2005;73:689-698.

9. Hintikka U, Laukkanen E, Marttunen M, Lehtonen J. Good working alliance and psychotherapy are associated with positive changes in cognitive performance among adolescent psychiatric inpatients. Bull Menninger Clin. 2006;70:316-335.

10. Ferrigno G, Marcenaro M, Penati S, et al. Un'esperienza psicoterapica ambulatoriale con adolescenti e genitori: Alcune considerazioni su una metodologia operativa. The Italian On Line Psychiatric Magazine. 2006. Availabe at: http://www.psychiatryonline.it/ital/adolescenti2009.htm. Accessed on April 14, 2010.

11. Hawley KM, Weisz JR. Youth versus parent working alliance in usual clinical care: Distinctive associations with retention, satisfaction, and treatment outcome. J Clin Child Adolesc Psychol. 2005;34: $117-128$.

12. Diamond G, Josephson A. Family-based treatment research: A 10-year update. J Am Acad Child Adolesc Psychiatry. 2005;44:872-887.

13. Kazdin AE, Whitley M, Marciano PL. Child-therapist and parent-therapist alliance and therapeutic change in the treatment of children referred for oppositional, aggressive, and antisocial behavior. J Child Psychol Psychiatry. 2006;47:436-445.

14. Nock MK, Ferriter C. Parent management of attendance and adherence in child and adolescent therapy: A conceptual and empirical review. Clin Child Fam Psychol Rev. 2005;8:149-166.

15. Nock MK, Kazdin AE. Randomized controlled trial of a brief intervention for increasing participation in parent management training. J Consult Clin Psychol. 2005;73:872-879.

16. Schaeffer CM, Borduin CM. Long-term follow-up to a randomized clinical trial of multisystemic therapy with serious and violent juvenile offenders. J Consult Clin Psychol. 2005;73:445-453.

17. Charmaine C,April C. Factors associated with insight among outpatients with serious mental illness. Psychiatr Serv. 2002;53:96-98.

18. Wittorf A, Jakobi U, Bechdolf A, et al. The influence of baseline symptoms and insight on the therapeutic alliance early in the treatment of schizophrenia. Eur Psychiatry. 2009;24:259-267. 
19. Lincoln TM, Lüllmann E, Rief W. Correlates and long-term consequences of poor insight in patients with schizophrenia. A systematic review. Schizophr Bull. 2007;33:1324-1342.

20. Marcelli D, Bracconier A. Adolescence et Psychopathologie. Paris, France: Masson; 1995.

21. Quintiliani R. The consultation with adolescents. 2007. Available from http://www.yahoo.acocms.it/print.asp?id=14408Roma. Accessed May 28, 2009.

22. Graafsma T, Anbeek M. Resistance in psychotherapy with adolescents. J Adolesc. 1984;7:1-16.

23. Frieswyk SH, Allen JG, Colson DB, et al. Therapeutic alliance: Its place as a process and outcome variable in dynamic psychotherapy research. J Couns Psychol. 1986;54:32-38.

24. Tay SE. Compliance therapy: An intervention to improve inpatients' attitudes toward treatment. J Psychosoc Nurs Ment Health Serv. 2007;45:29-37.

25. Laufer M. Adolescent Breakdown and Beyond. London, UK: Karnak Books; 1997.

26. Wade TD, Frayne A, Edwards SA, Robertson T, Gilchrist P. Motivational change in an inpatient anorexia nervosa population and implications for treatment. Aust N Z J Psychiatry. 2009;43(3):235-243.

27. Gatta M, Giovanatto A, Condini A. Clinical activities of a centre for adolescents' psychopathology: A longitudinal study. Ital J Child Adolesc Psychopathol Psychiatry. 2003;2:107-120.

28. Martin DJ, Garske JP, Davis MK. Relation of the therapeutic alliance with outcome and other variables: A meta-analytic review. J Consult Clin Psychol. 2000;68:438-450.

29. Kemp R, David A. Insight and compliance. In: Blackwell B, editor. Treatment Compliance and Therapeutic Alliance. Amsterdam, The Netherlands: Harwood Academic Publishers; 1997.

30. Leichsenring F, Leibing E. Psychodynamic psychotherapy: A systematic review of techniques, indications and empirical evidence. Psychol Psychother. 2007;80:217-228.

31. Markova IS, Berrios GE. The assessment of insight in clinical psychiatry: A new scale. Acta Psychiatr Scand. 1992;86:159-164.

32. Wechsler D. WISC-R Wechsler Intelligent Scale for Children Revised. In: Rubini V, Padovani F, editors. Manuale, Traduzione e Adattamento Italiano. Firenze, Italy: Organizzazioni Speciali; 1986.

33. Wechsler D. WAIS-R Wechsler Adult Intelligence Scale Revised. In: Laicardi C, Orsini A, editors. Manuale, Traduzione e Adattamento Italiano. Firenze: Organizzazioni Speciali; 1997.

34. World Health Organization. The ICD-10 Classification of Mental and Behavioural Disorders: Clinical Descriptions and Diagnostic Guidelines. Geneva, Switzerland: WHO. Trad. It. Milano: Masson; 1992.
35. Roncone R, Tozzini C, Mazza M, De Risio A, Morosini P, Casacchia M. Validation of the Italian version of the self-report Insight Scale. Epidemiol Psichiatr Soc. 2003;12:63-75.

36. Birchwood M, Smith J, Drury V, Healy J, Macmillan F, Slade M. A self report insight scale for psychosis: Reliability, validity and sensitivity to change. Acta Psychiatr Scand. 1994;89:62-67.

37. Conti L. IS Insight Scale. In: Repertorio delle Schede di Valutazione in Psichiatria. Firenze, Italy: SEE; 2002.

38. Horvath AO, Greenberg LS. Development and Validation of the Working Alliance Inventory. The entity from which ERIC acquires the content, including journal, organization, and conference names, or by means of online submission from the author. J Couns Psychol. 1989;36:223-233.

39. Di Giuseppe R, Linscott J, Jilton R. Developing the therapeutic alliance in children-adolescent psychotherapy. Appl Prev Psychol. 1996;5: 85-100.

40. Lingiardi V. L'Alleanza Terapeutica. Teoria, Clinica, Ricerca. Milan, Italy: Raffaello Cortina; 2002.

41. Bordin ES. The generalizability of the psychoanalytic concept of the working alliance. Psychotherapy: Theory, Research and Practice. 1979; 16:252-260

42. Horvath AO. Empirical validation of Bordin's pantheoretical model of the alliance: The Working Alliance Inventory perspective. In Horvath AO, Greenberg LS, editors. The Working Alliance: Theory, Research, and Practice. New York, NY: Wiley; 1994:109-128.

43. Hanson WE, Curry KT, Bandalos DL. Reliability generalization of Working Alliance Inventory Scale Scores. Educ Psychol Meas. 2002;62(4):659-673.

44. Aliprandi M, Pelanda E, Senise T. Psicoterapia Breve di Individuazione. Milan: Feltrinelli; 1999.

45. Derksen JJ. Short-term psychodynamic psychotherapy. Tijdschr Psychiatr. 2006;48(10):777-786.

46. Conti L. CGI Clinical Global Impression. In: Repertorio delle Schede di Valutazione in Psichiatria. Firenze: SEE; 2002

47. Department of Health and Children. Working Group on Child and Adolescent Psychiatric Services: Second report June 2003. Retrieved from http://www.lenus.ie/hse/bitstream/10147/46353/1/1294.pdf. Accessed May 28, 2009.

48. Luborsky L, Mintz J, Auerbach A, et al. Predicting the outcome of psychotherapy. Findings of the Penn Psychotherapy project. Arch Gen Psychiatry. 1980;37:471-481

49. Gaston L, Thompson L, Gallagher D, Cournoyer LG, Gagnon R. Alliance, technique and their interactions in predicting outcome of behavioural, cognitive and brief dynamic therapy. Psychother Res. 1998;8:190-209.
Adolescent Health, Medicine and Therapeutics

\section{Publish your work in this journal}

Adolescent Health, Medicine and Therapeutics is an international, peer-reviewed, open access journal focusing on health, pathology, and treatment issues specific to the adolescent age group. All aspects of health maintenance, preventative measures and disease treatment interventions are addressed within the journal and practitioners from

\section{Dovepress}

all disciplines are invited to submit their work as well as healthcare researchers and patient support groups.. The manuscript management system is completely online and includes a very quick and fair peerreview system. Visit http://www.dovepress.com/testimonials.php to read real quotes from published authors. 